\section{An epidemiological study of 97 cases of primary cicatricial alopecia in Iran}

\author{
Akram Beheshtiroy, ${ }^{1}$ Fatemeh \\ Hajmanoochehri, ${ }^{2}$ Fahim Hossienghamar ${ }^{1}$ \\ Departments of ${ }^{1}$ Dermatology and \\ 2Pathology, Qazvin University of Medical \\ Sciences, Iran
}

disease could be stopped through early and correct diagnosis. ${ }^{1}$

Previous research on PCA is sparse, particularly from Asia. ${ }^{4,5}$ The predominant histological type was lymphocytic in some studies and neutrophilic in some other studies. ${ }^{3,6-8}$

The present study aimed to examine the epidemiological features of PCA cases in a university hospital in Iran.

\section{Materials and Methods}

\section{Abstract}

Cicatricial alopecia can produce major psychosocial disturbance. This study aimed to evaluate epidemiological features of primary cicatricial alopecia (PCA). Epidemiological and histological types of scarring alopecia were determined. The chi-squared test and $t$-test were performed to examine the relationship between different variables using SPSS 16. A total of 97 cases, 35 (36.1\%) men and 62 (63.9\%) women, with an average age of 37 $(\mathrm{SD}=12.7)$, were included in this study. The female/male (F/M) ratio was 1.7:1 for total cases, 1.92:1 for the lymphocytic type, and 1:1.5 for the neutrophilic type. No relationship was found between type of job (indoor or outdoor), clinical findings and histological types. Discoid lupus erythematous (DLE) was the most common histological type (63.9\%). PCA was found to mainly affect middle-aged individuals. The high percentage of DLE in the present study is in agreement with some similar studies.

\section{Introduction}

Cicatricial or scarring alopecia is defined as permanent loss of hair follicles, and its hallmarks are the loss of follicular ostia in clinical examination and destruction of hair follicle in histological examination. ${ }^{1}$ Cicatricial alopecia can produce some disfigurement and psychosocial disturbances. ${ }^{2}$ It is divided into primary and secondary types. In primary cicatricial alopecia (PCA), the hair follicle itself is the target for inflammation and destruction, whereas in the secondary type, pathological changes begin from non-follicular dermis., ${ }^{1,3}$

Histopathology of scalp is necessary in distinguishing scarring alopecia from the nonscarring type. Also, based on histopathological patterns, PCA is divided into lymphocytic, neutrophilic, mixed, and nonspecific types. ${ }^{1}$ This classification is important because different treatment strategies may be used depending on the histological type of PCA. ${ }^{4}$ Although there is no hope for regrowth of destructed hair, there is a chance that the progression of the
All cases of histopathological PCA diagnosed over a period of ten years in the skin ward and pathology department of a university hospital in Iran were included in our study. Age, sex, type of job (indoor or outdoor), and clinical findings were determined from hospital records of the patients. Histological examination was performed on 4-mm skin specimens sectioned horizontally and vertically and stained with hematoxylin and eosin. Also, special staining was performed every time it was needed.

Histological types of PCA were determined according to pathology reports. Based on the classification provided in 2001 by the North American Hair Research Society, discoid lupus erythematous (DLE), lichen planopilaris (LPP) and its variants, and pseudopelade of Brocq (PPB) were regarded as lymphocytic PCA. Folliculitis decalvans (FD) and dissecting cellulitis/folliculitis (DCF) were regarded as neutrophilic PCA. Folliculitis keloidalis (as a subclass of mixed PCA) and nonspecific PCA were collectively considered other types.

The data were described using descriptive statistics (frequency, mean, and standard deviation). The relationship between different variables was analyzed using the chi-squared test and $t$-test on SPSS 16 (IBM Corporation, Armonk, NY, USA, 2008). The significance level was set at $\mathrm{P}<0.05$.

\section{Results}

A total of 97 cases, 35 (36.1\%) men and 62 (63.9\%) women, were included in this study. The patients' age ranged from 10 to 70 years, with an average age of $37(\mathrm{SD}=12.7)$. As Table 1 shows, most patients (32\%) were in the 40 to 49 age group, and no relationship was found between age group and histological type $(\mathrm{P}=0.97)$. It can be seen from Table 1 that most cases (84.5\%) were of the lymphocytic type. Also, in the neutrophilic group, there were nine cases of FD and only one case of DCF. Furthermore, of the cases under the other heading, four were nonspecific PCA, and one was of the folliculitis keloidalis type.
Correspondence: Fatemeh Hajmanoochehri, Department of Pathology, Qazvin University of Medical Sciences, Shahid Bahonar Blvd, Qazvin, Iran.

Tel.Fax: +98.283 .332 .4970 .

E-mail: f.manoochehri@yahoo.com

Key words: Cicatricial alopecia; histology; epidemiology.

Contributions: the authors contributed equally.

Conflict of interest: the authors declare no potential conflict of interest.

Received for publication: 20 April 2015.

Revision received: 2 June 2015.

Accepted for publication: 12 June 2015.

This work is licensed under a Creative Commons Attribution NonCommercial 3.0 License (CC BYNC 3.0).

(C) Copyright A. Beheshtiroy et al., 2015

Licensee PAGEPress, Italy

Dermatology Reports 2015; 7:5960

doi:10.4081/dr.2015.5960

The female/male (F/M) ratio was 1.7:1 for total cases. This ratio was 1.9:1 for the lymphocytic category, 1:1.5 for the neutrophilic group, and 4:1 for the other category. No relationship was observed between sex and type of job on the one hand and histological type of alopecia on the other (P-values were 0.48 and 0.94, respectively). Itching, scalp tenderness, and ulceration were common clinical problems, but no relationship was seen between these problems and histological diagnosis (P-values were $0.45,0.72$, and 0.07 , respectively).

\section{Discussion}

There is a paucity of data on the epidemiology of PCA. ${ }^{9}$ Table 2 gives a summary of the results obtained in the present study and a few other similar studies. ${ }^{5-8}$

A wide range of patient age was seen in the present research, but the disease was more common among the middle-aged individuals. This finding is close to the results reported by similar studies. ${ }^{3-7}$

Another finding was that PCA affected women about 1.7 times more than it affected men. An almost double dominance has also been reported for female patients in some other studies. ${ }^{3,5,6,9}$ In contrast, a study in Iran showed that PCA affected male patients more commonly. ${ }^{8}$ This inconsistency can be attributed to the fact that a high percentage of PCA cases in this reference were of the neutrophilic type. Indeed, past research has shown 
that neutrophilic PCA is more common in middle-aged men, but lymphocytic PCA is more frequently seen among middle-aged women. ${ }^{10}$ Another point to make is that the highest F/M ratio in our study concerned the PPB type. Similar F/M ratios have also been reported in some other studies. ${ }^{4,6}$

In our study, no relationship was seen between the type of job and histological types of PCA. However, a study in Iraq found that most male patients had outdoor jobs, and the author ascribed their alopecia to the possible effect of some environmental factors such as exposure to sunlight. ${ }^{5}$ Indeed, sun exposure has been shown to play a role in developing some types of PCA such as DLE and LPP. ${ }^{1,3}$

As reported in another study, itching, scalp tenderness, and ulceration were commonly seen in our patients, but no relationship was found between these clinical problems and histological types of PCA. ${ }^{5}$

In this study, DLE turned out to be the most common histological type, a finding which is in agreement with some other studies.,5,10,11 However, there are studies which reported FD and PPB as the most common type. ${ }^{3,7,8}$ Another observation in the present study was that the ratio of lymphocytic PCA to neutrophilic/other types of PCA was 5.5:1. This ratio was 7.4:1, 8.5: $1,0.78: 1$, and $1.86: 1$ in other studies..$^{5-8}$ This inconsistency is probably multifactorial, with a possible factor being the process whereby patients are selected for biopsy. For example, it is less common to perform biopsy for FD and DCF if the distinctive clinical and dermatoscopic features are present. ${ }^{7}$ In our study and also in some other studies, neutrophilic type of PCA makes up a small percentage of PCA. ${ }^{5,6}$

Non-specific PCA constituted a low percentage of histological types unlike in the study done by Whiting. ${ }^{3}$ This difference may be due to the fact that patients refer to a dermatologist at a low rate when the disease subsides or because the clinician tends to choose not to do biopsy at this stage. Our knowledge of PCA etiology is low, but different etiological factors such as infectious agents, drug reaction, or sun exposure may have a role in developing PCA. ${ }^{1}$ Frequency of etiological factors in different populations maybe another contributor to different percentages reported for different histological types. Also, genetic or ethnic factors may have an importance role. ${ }^{12}$

A limitation of this study is that it only included those PCA cases for which biopsy had been performed. This decision was made due to the retrospective nature of the present study, which investigated the hospital evidence amassed over a ten-year-long period. Unfortunately, we realized that the recording system of the hospital was rather imperfect. It might as well be noted that the studies cited in this work have similarly dealt with the biopsied cases only. Before we bring this paper to an end, it is advisable to give a brief account of the treatment options available for PCA. Delayed or incorrect diagnosis of the diseases affecting hair follicles leads to permanent follicular loss. ${ }^{1,9}$ Classification of PCA into lymphocytic, neutrophilic, and mixed categories has the advantage that, although each specific histological type of PCA requires a special treatment option, there are general rules for the treatment of the PCA cases within each of the three main categories. ${ }^{4,9}$ More specifically, the general treatment for the lymphocytic cases of PCA is to reduce inflammation by means of immunosuppression or through the use of immune system modulating agents. On the other hand, the main treatment decision for the neutrophilic cases of PCA is to eliminate microbes through the use of antimicrobial agents. And finally, mixed treatment could be administered for the cases within the mixed category. ${ }^{4,13}$ Immunosuppression is usually performed via some oral medications such as oral corticosteroids and cyclosporine or through the use of local drugs such as intraleisional triamcinolone acetonide or the betamethasone dipropionate topical cream. Furthermore, two main immunomodulators are hydroxychloroquine and thalidomide. .,9,13 $^{4}$

As for the neutrophilic cases of PCA, the recommended treatment is the culture of hair follicle pustule or the administration of antibiotics according to the antibiotic sensitivity report. For this purpose, antibiotic agents such as cloxacillin, clindamycin, rifampicin, tetracycline, and dapson could be used individually or in combination with an anti-inflammatory drug such as colchicine. ${ }^{4,9,13}$

\section{Conclusions}

This study reviewed significant cases of his-

Table 1. The relationship between age groups and histological types of alopecia $(P=0.97)$.

\begin{tabular}{lcccccc} 
& DLE & $\begin{array}{c}\text { Lymphocytic } \\
\text { LPP }\end{array}$ & PPB & Neutrophilic & Other & Total (\%) \\
$10-19$ & 6 & 1 & 1 & 1 & 1 & $10(10.3)$ \\
$20-29$ & 10 & 2 & 2 & 2 & 1 & $17(17.5)$ \\
\hline $30-39$ & 16 & 2 & 2 & 4 & 0 & $24(24.7)$ \\
$40-49$ & 21 & 6 & 1 & 1 & 2 & $31(32.0)$ \\
\hline $50-59$ & 6 & 2 & 0 & 2 & 1 & $11(11.3)$ \\
$60-69$ & 2 & 1 & 0 & 0 & 0 & $3(3.1)$ \\
\hline $70-79$ & 1 & 0 & 0 & 0 & 0 & $1(1.0)$ \\
Total (\%) & $62(63.9)$ & $14(14.4)$ & $6(6.2)$ & $10(10.3)$ & $5(5.2)$ & $97(100)$ \\
\hline
\end{tabular}

DLE, discoid lupus erythematous; LPP, lichen planopilaris; PPB, pseudopelade of Brocq.

Table 2. A comparison between the findings of the present study and those of a few similar studies.

\begin{tabular}{|c|c|c|c|c|c|c|c|c|c|c|c|c|}
\hline \multirow[t]{2}{*}{ Studies } & \multicolumn{6}{|c|}{ Lymphocytic } & \multicolumn{2}{|c|}{ Neutrophilic } & \multicolumn{2}{|c|}{ Other } & \multicolumn{2}{|c|}{ Total } \\
\hline & No $(\%)$ & F:M & No (\%) & F:M & № (\%) & F:M & No $(\%)$ & F:M & No $(\%)$ & F:M & No & F:M \\
\hline This study & $62(63.9)$ & 1.8:1 & 14 (14.4) & $1.8: 1$ & $6(6.2)$ & $5: 1$ & $10(10.3)$ & 1:1.5 & $5(5.2)$ & $4: 1$ & 97 & $1.7: 1$ \\
\hline Moure et al. ${ }^{6}$ & $17(43.6)$ & $4.7: 1$ & $4(10.2)$ & $1: 1$ & $12(30.8)$ & $5: 1$ & $4(10.3)$ & $1: 1$ & $2(5.1)$ & $1: 1$ & 39 & $2.8: 1$ \\
\hline Al-Hilo et al. ${ }^{5}$ & $17(40.5)$ & $4.6: 1$ & $12(28.6)$ & $2: 1$ & $8(19)$ & $1.6: 1$ & $3(7.1)$ & $1: 2$ & $2(4.8)$ & $0: 2$ & 42 & $2: 1$ \\
\hline Nejad et al. ${ }^{8}$ & $25(25)$ & $1.5: 1$ & $14(14)$ & $2.5: 1$ & $18(18)$ & $2: 1$ & $30(30)$ & $0.25: 1$ & $13(13)^{*}$ & $0.62: 1$ & 100 & $0.9: 1$ \\
\hline $\mathrm{Qi}^{7}$ & $13(22)$ & $5.5: 1$ & $2(3.4)$ & $1: 1$ & $9(15.3)$ & $0.8: 1$ & $33(55.9)$ & $0.3: 1$ & $2(3.4)^{\circ}$ & $1: 10$ & 59 & $1.6: 1$ \\
\hline
\end{tabular}

DLE, discoid lupus erythematous; LPP, lichen planopilaris; PPB, pseudopelade of Brocq.*Including eight cases of morphea (secondary type of scarring alopecia). ${ }^{\circ}$ Two cases of alopecia mucinosa were in this group. 
tologically confirmed PCA obtained over a period of ten years. The ratio of lymphocytic alopecia to neutrophilic/mixed alopecia or other types was 5.5:1. PCA was found to be more common among middle-aged individuals, and it had a tendency to affect middle-aged women in the case of the lymphocytic type. Also, the neutrophilic type was more common among middle-aged men. Itching, scalp tenderness, and ulceration were common in our patients. No relationship was observed between clinical findings, type of job (indoor or outdoor), and histological types. More studies seem warranted to clarify the pathogenesis of PCA.

\section{References}

1. Elizabeth K, Ross EK, Tan E, Shapiro J. Update on primary cicatricial alopecia. J Am Acad Dermatol 2005;53:1-37.
2. Pradhan P, D'Souza M, Bade BA, et al. Psychosocial impact of cicatricial alopecia. Indian J Dermatol 2011;56:684-8.

3. Whiting DA. Cicatricial alopecia: clinicopathological findings and treatment. Clin Dermatol 2001;19:211-25.

4. Kumar UM, Yelikar BR. The spectrum of histopathological lesions in scarring alopecia: a prospective study. J Clin Diagn Res 2013;7:1372-6.

5. Al-Hilo MM, Al-Saedy SJ, Yacoob PY. Primary cicatricial alopecia: a clinical and histopathological descriptive study. Am J Dermatol Venereol 2013;2:15-22.

6. Moure ER, Romiti R, Machado MC, Valente NY. Primary cicatricial alopecias: a review of histopathologic findings in 38 patients from a clinical university hospital in Sao Paulo, Brazil. Clinics (Sao Paulo) 2008;63:747-52.

7. Qi S, Zhao Y, Zhang X, et al. Clinical features of primary cicatricial alopecia in
Chinese patients. Indian $\mathrm{J}$ Dermatol Venereol Leprol 2014;80:306-12.

8. Nejad SB, Khodaeiani E, Amirinia M, Goldust M. Evaluation of cicatricial alopecia in Iran. Pak J Biol Sci 2013;16;1609-11.

9. Dogra S, Sarangal R. What's new in cicatricial alopecia? Indian J Dermatol Venereol Leprol 2013;79:576-90.

10. Tan E, Martinka M, Ball N, Shapiro J. Primary cicatricial alopecias: clinicopathology of 112 cases. J Am Acad Dermatol 2004;50:25-32.

11. Inchara YK, Tirumalae R, Kavdia R, Antony M. Histopathology of scarring alopecia in Indian patients. Am J Dermatopathol 2011;33:461-7.

12. Harries MJ, Paus R. The pathogenesis of primary cicatricial alopecias. Am J Pathol 2010;177:2152-62.

13. Abal-Díaz L, Soria X, Casanova-Seuma JM. [Scarring alopecia]. Actas Dermosifiliogr 2012; 103:376-87. [Article in Spanish]. 